\title{
The Legacy of LEP B Program
}

\author{
Yoram Rozen
}

Physics Department

Technion, Israel Institute of Technology

- The PreLEP B world

- Expectation from the LEP B program

- Major modification

- LEP B program - results

- Comments, future, summary 


\section{The PreLEP B world}

- Active players:

ARGUS

CLEO1 (1.5)

- Spectroscopy:

Fairly well established $\Upsilon$ states (1-5).

Only $B_{u}$ and $B_{d}$ were seen.

- Branching fractions - Only $9 B_{u}$ exclusive modes were measured with a total under $7 \%(<3 \%)$. Only $b \rightarrow c$ decays were measured*.

- Rare decays - of-course (if you call $10^{-3}$ rare)

- CKM elements:

$$
\begin{aligned}
& V_{c b}=0.044 \pm 0.009 \\
& V_{u b}=0.004 \pm 0.002 \text { mostly sl decays with }
\end{aligned}
$$

one controversial non leptonic evidence.

- B mixing: time integrated - leading to

$$
\Delta m=4.0 \pm 0.8 \times 10^{-10} \mathrm{MeV}
$$

- B lifetime: $1.18 \pm 0.11$ ps (from non- $\Upsilon$ machines) 
The expected LEP B program

- Lifetime

2.6.2. EXPERIMENTAL ASPECTS*

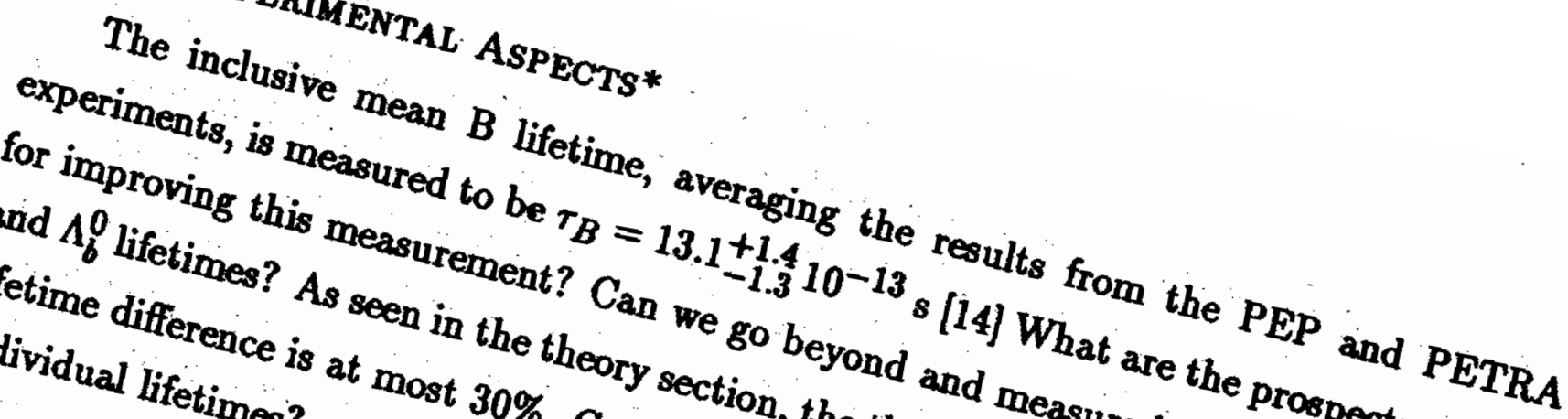
for improving this measurement? $C_{a n}=13.1+1.410-13$ se $[14]$ Who beyond and wat are the prospects at LEP and $A_{b}^{0}$ lifetimes? As seen in the theory we go beyond and measure individual $B_{d}^{0}, B_{t}^{+}, B_{s}^{0}$

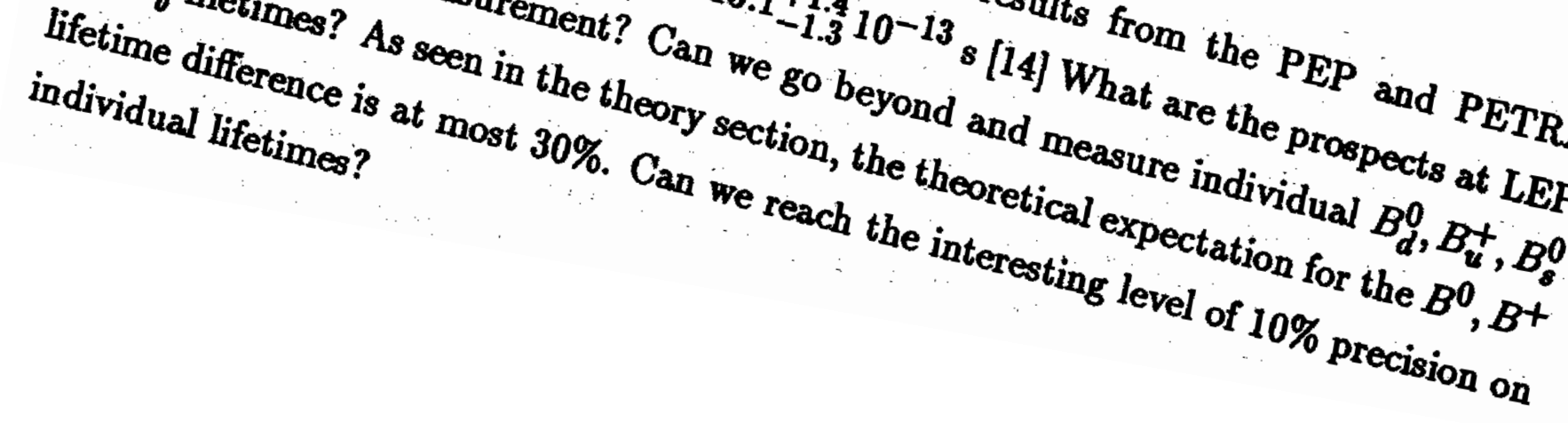

Y. Bozen

June. 2002 


\section{- B mixing}

Time integrated methods:

1. b-b expected precision on $\bar{\chi}$ of $15 \%$ for $10^{6} Z^{0}$

2. B-b with $10^{7} Z^{0}$ LEP expected $25 \%$ statistical accuracy on $\chi_{s}$

3. B-B not clear if possible even with $10^{7} Z^{0}$

Time dependent: expecting to confirm $B_{d}$ time evolution of mixing - no prediction for error size.

\section{- CKM}

A conservative estimate for $\left|V_{u b} / V_{c b}\right|$ was expecting an upper limit below 0.21 . 


\section{Remembering LEP}

- First collision - Aug. 1989

- Last collision - Nov. 2000

- $175 \mathrm{pb}^{-1}$ or $4.4 \mathrm{M}$ hadronic events/exp around $91 \mathrm{GeV}$

- $725 \mathrm{pb}^{-1}$ between 130 and $210 \mathrm{GeV}$

- 4 Detectors:

\begin{tabular}{|l|c|c|c|c|}
\hline Parameter & ALEPH & DELPHI & L3 & OPAL \\
\hline Tracking & TPC & TPC & small drift ch. & Drift ch. (4 bar) \\
Calorimetry & lead/wire ch. & HPC & BGO & lead-glass \\
Particle ID & $\mathrm{dE} / \mathrm{dx}$ & $\mathrm{RICH}$ & $\mathrm{e} / \mu$ & $\mathrm{dE} / \mathrm{dx}$ \\
Magnet & $1.5 \mathrm{~T}$ & $1.2 \mathrm{~T}$ & $0.5 \mathrm{~T}$ & $0.4 \mathrm{~T}$ \\
Vertexing & Si 89,91 & Si $89,91,94$ & $\mathrm{Si} 93$ & Si 9193 \\
\hline
\end{tabular}




\section{Improved Vertexing - The B Program Booster}

With an average momentum of $25 \mathrm{GeV}$ a $\mathrm{B}$ meson travels an average of $2.5 \mathrm{~mm}$.

It was quickly realized that a precise vertex determination will dramatically improve the B program.

All 4 detectors armed themselves with SVDs.

\section{Performance:}

ALEPH - IP resolution of $25 \mu m$ in both $r-\phi$ and $z$.

DELPHI - IP resolution of $\sqrt{\left(69 / p_{t}\right)^{2}+24^{2}}$ (9.5).

L3 - single track resolution of 7 and $14 \mu \mathrm{m}$.

OPAL - IP resolution of $18-24 \mu \mathrm{m}$. 


\section{LEP B Physics - Results}

- Lifetime.

1. $B_{d}^{0}$

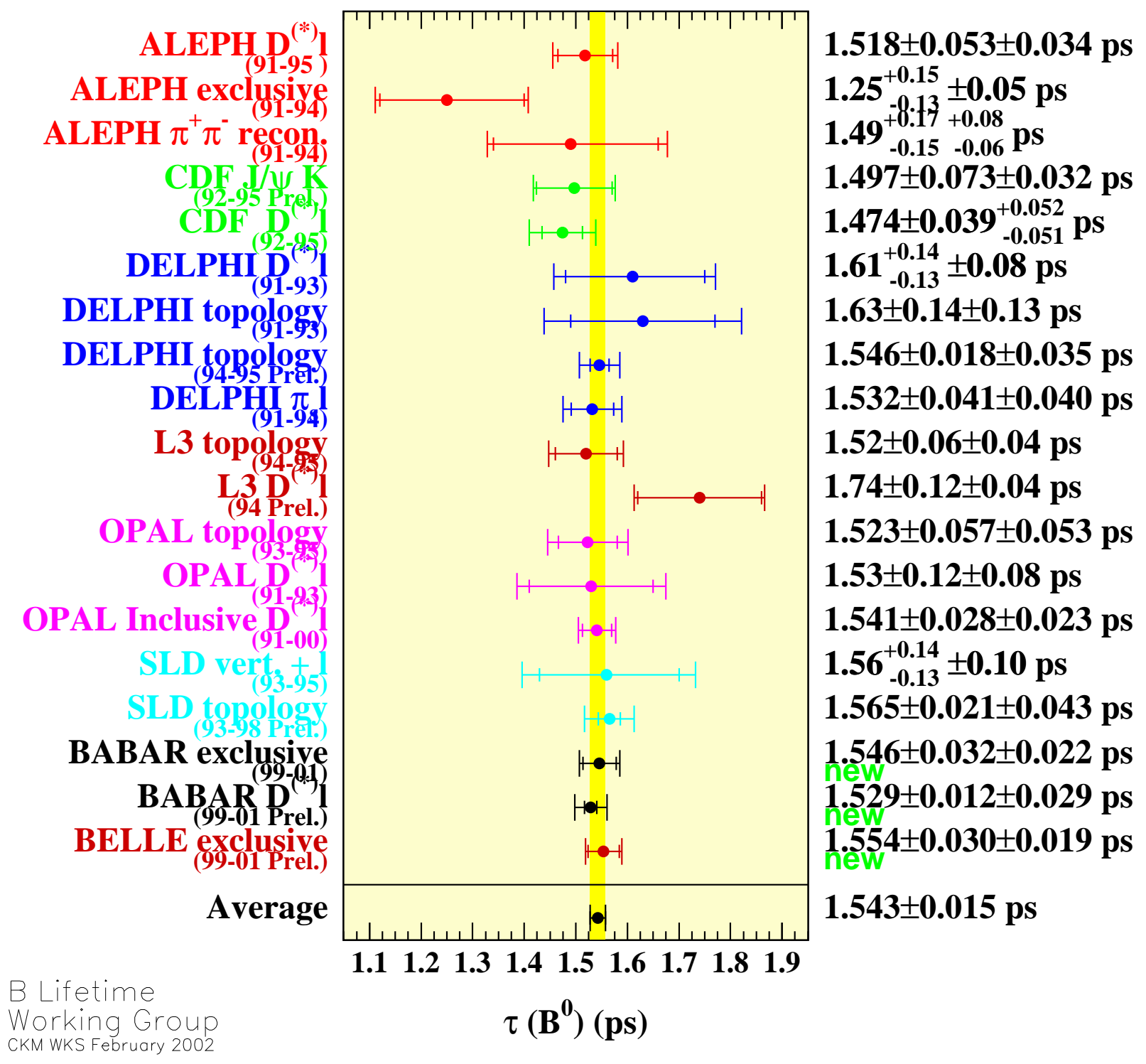


2. $B_{u}^{-}$

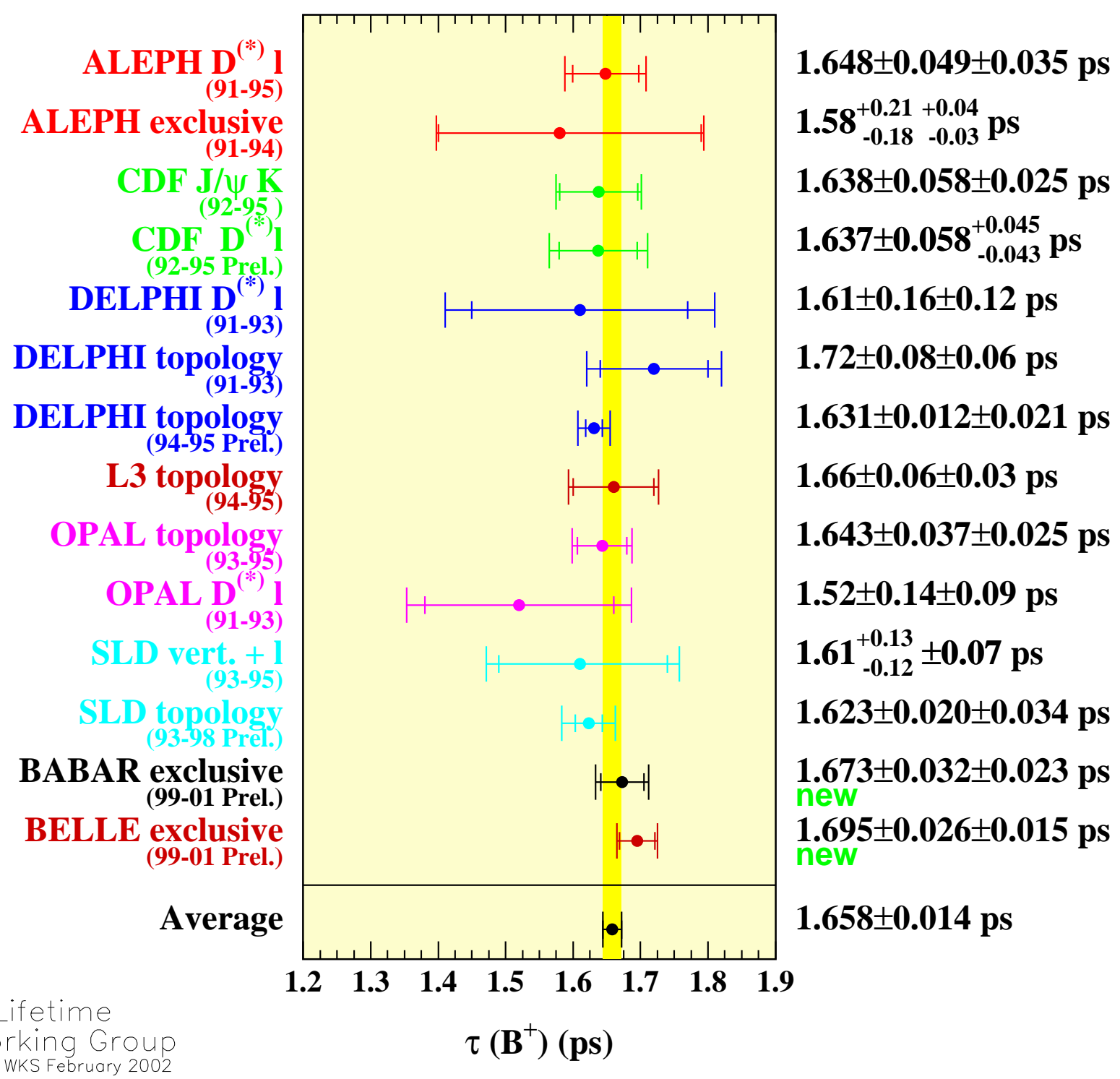


3. Direct measurements of the Ratio $\tau_{B_{u}^{-}} / \tau_{B_{d}^{0}}$

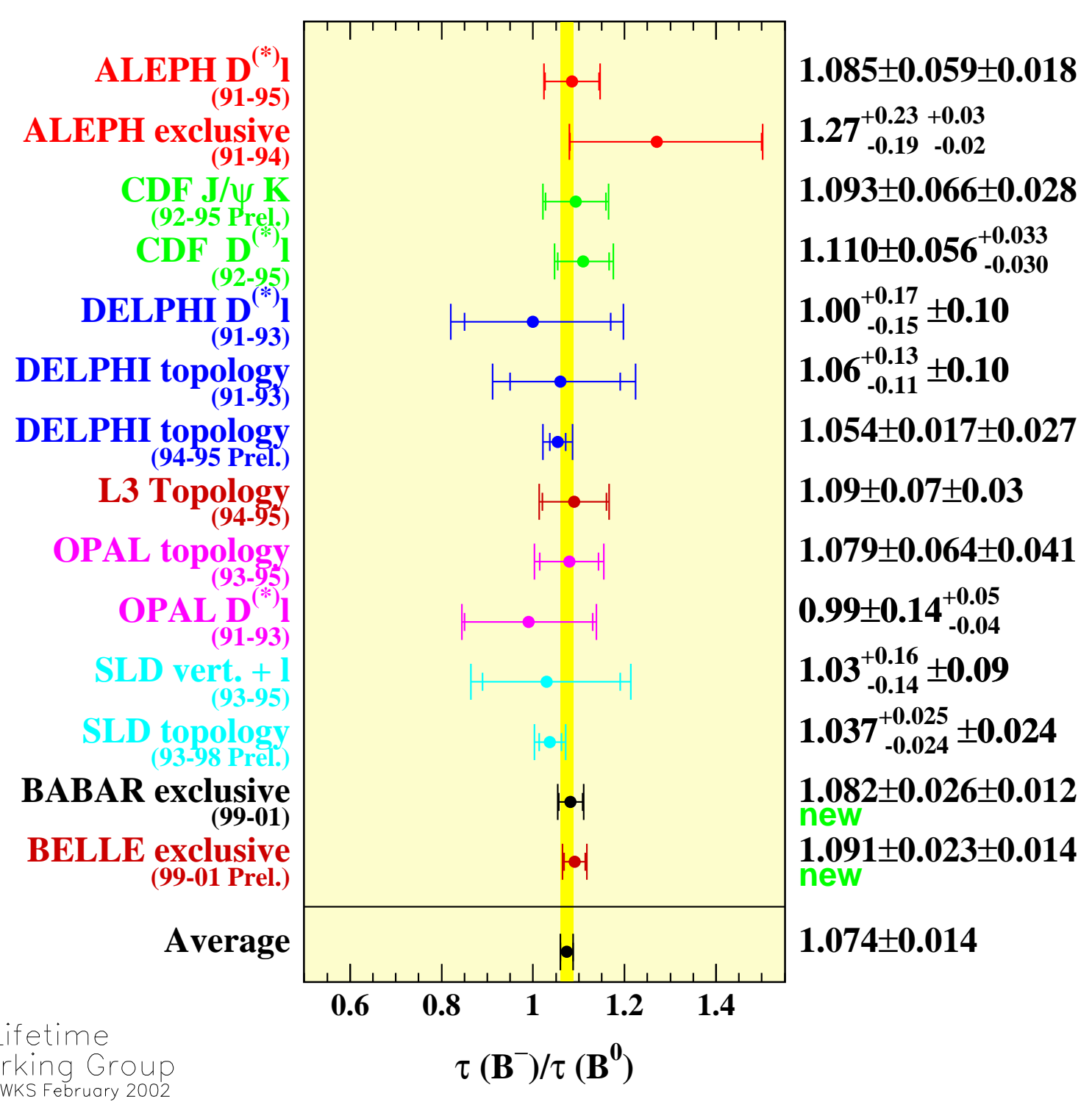


4. $B_{s}^{0}$

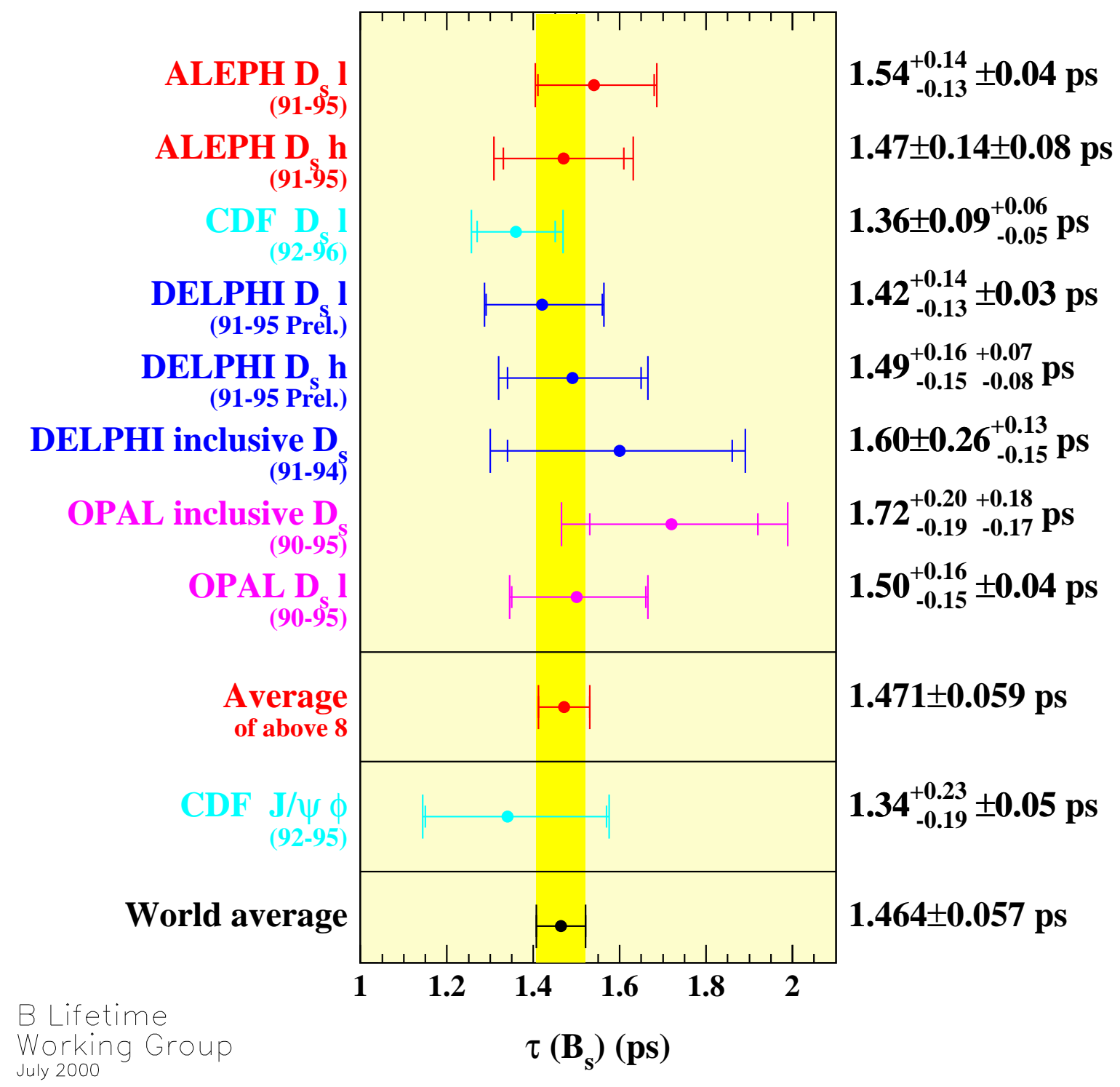


5. $\Lambda_{b}$

Avg b baryon meas.

ALEPH $\Lambda$ I

(91-95)

DELPHI p I (91-95)

DELPHI $\Lambda \mathrm{l} \pi$ (91-95)

DELPHI $\Lambda$ I

(91-95)

OPAL $\Lambda$ I (IP+vtx)

(90-94)

$\Lambda_{b}$ measurements

ALEPH $\Lambda_{c} 1$

(91-95)

ALEPH $\Lambda \mathrm{l}^{+} \mathrm{l}^{-}$

(91-95)

$\mathrm{CDF} \Lambda_{c} 1$

(92-95)

DELPHI $\Lambda_{c} \mathrm{l}$

(91-95)

OPAL $\Lambda_{c} 1$

Average of above 10

\section{$\Xi$ l measurements}

ALEPH $\Xi 1$

(90-95)

DELPHI $\Xi 1$

(91-93)

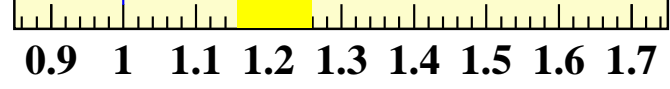

$1.20 \pm 0.08 \pm 0.06 \mathrm{ps}$

$1.19 \pm 0.14 \pm 0.07 \mathrm{ps}$

$1.16 \pm 0.20 \pm 0.08 \mathrm{ps}$

$1.10_{-0.17}^{+0.19} \pm 0.09$ ps

$1.16 \pm 0.11 \pm 0.06 \mathrm{ps}$

$1.18_{-0.12}^{+0.13} \pm 0.03$ pS

$1.30_{-0.21}^{+0.26} \pm 0.04 \mathrm{ps}$

$1.32 \pm 0.15 \pm 0.06$ ps

$1_{-0.18}^{+0.19} \pm 0.05$ ps

$1_{-0.22}^{+0.24} \pm 0.06 \mathrm{ps}$

$1.208 \pm 0.051 \mathrm{ps}$

$1.35_{-0.28}^{+0.37+0.17} \mathrm{ps}$

$1.5_{-0.4}^{+0.7} \pm 0.3 \mathrm{ps}$
B Lifetime

Working Group

July 2000

$$
\tau \text { (b-baryon) (ps) }
$$


6. Comparison with theory

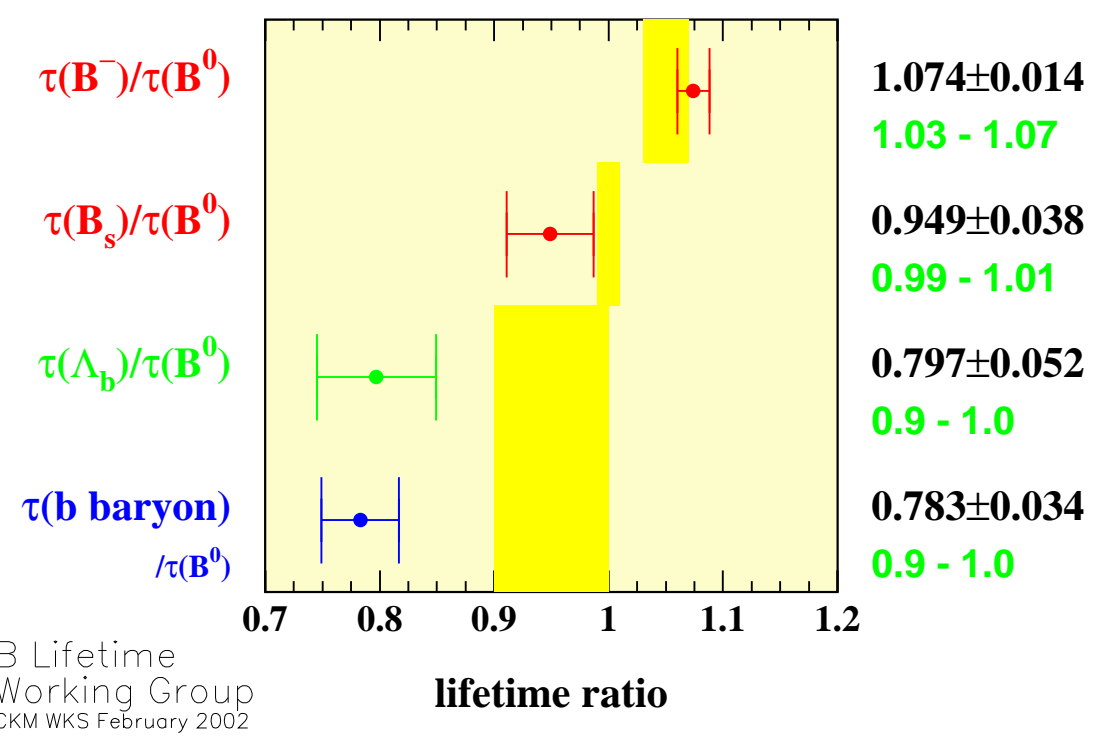

7. Lifetime summary
- $\tau_{B_{d}^{0}}=1.543 \pm 0.015 p s$
- $\tau_{B_{u}^{-}}=1.658 \pm 0.014 p s$
- $\tau_{B_{s}^{0}}=1.464 \pm 0.057 p s$
- $\tau_{\Lambda_{b}}=1.208 \pm 0.051 p s$ 
- $V_{u b}$

1. Find $\operatorname{Br}\left(b \rightarrow X_{u} l \nu\right)$

\section{Example - DELPHI}

- Hadronic mass of $X_{u}$ is expected to be smaller than that of $D$.

- Lepton IP w.r.t the secondary vertex should be negative for $b \rightarrow c$.
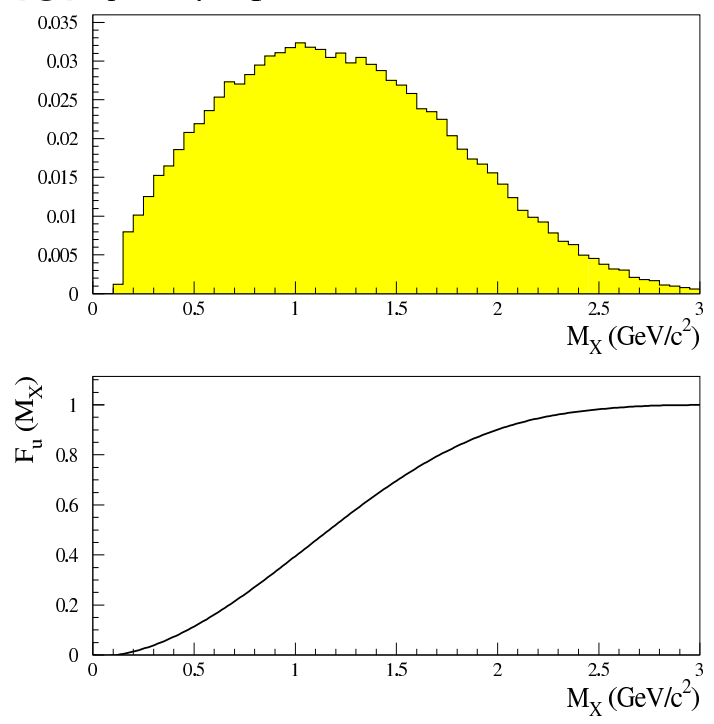

2. Use the connection

$\left|V_{u b}\right|=$

$0.00445\left[\frac{B R\left(b \rightarrow X_{u} l \nu\right)}{0.002} \frac{1.55}{\tau_{b}}\right]^{1 / 2}\left[1 \pm 2 \%\right.$ pert $\left.\pm 3.5 \% m_{b}\right]$ 


\section{Obtain:}

$$
\left|V_{u b}\right|=\left(4.09_{-0.69}^{+0.59}\right) \times 10^{-3}
$$

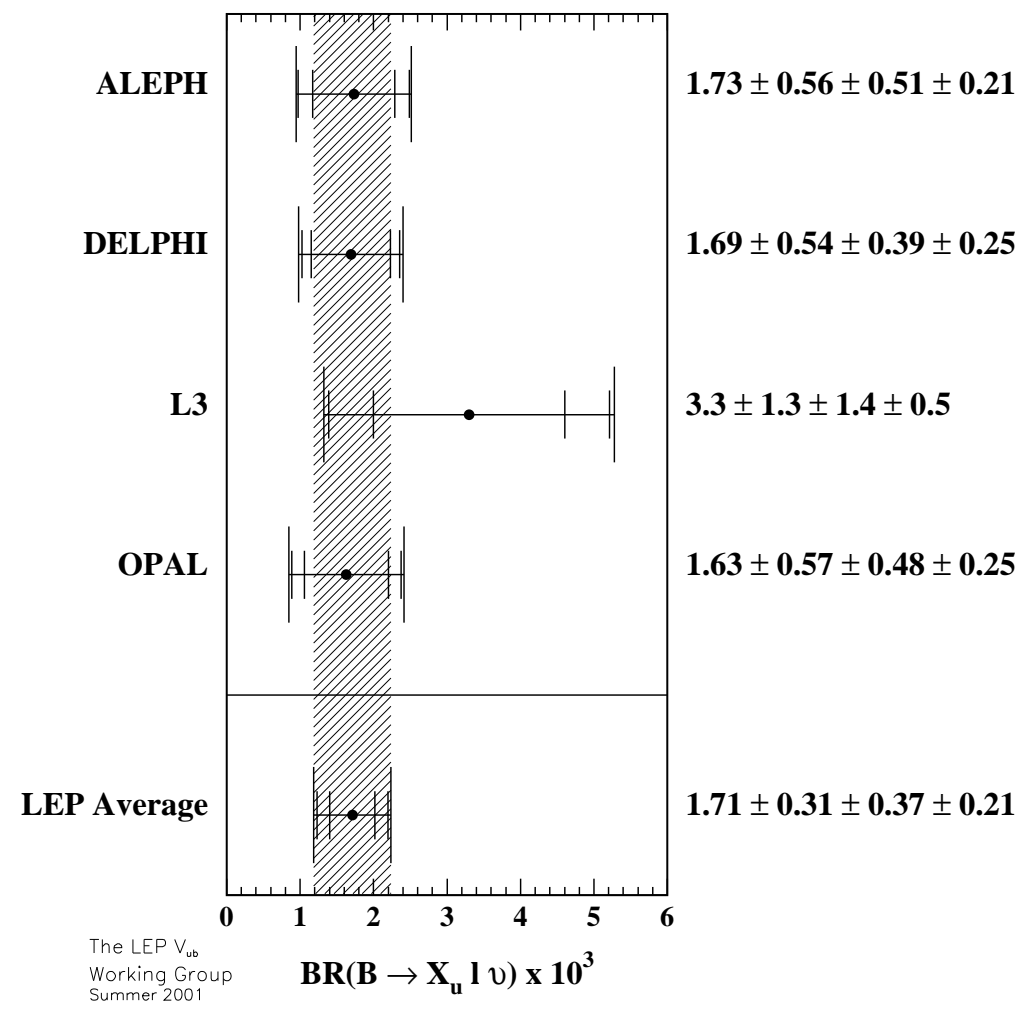


- $V_{c b}$

Method 1 - using $B^{0} \rightarrow D^{*+} l^{-} \nu$

Measure the decay rate as a function of $\omega$

$$
\omega=\frac{m_{D^{*}}^{2}+m_{B}^{2}-q^{2}}{2 m_{D^{*}} m_{B}}
$$

and use the connection:

$$
\frac{d \Gamma\left(B^{0} \rightarrow D^{*+} l^{-} \nu\right)}{d \omega}=\mathcal{K}(\omega) \mathcal{F}^{2}(\omega)\left|V_{c b}\right|^{2}
$$

\section{Example - OPAL}

Using $D^{0} \rightarrow K^{-} \pi^{+}, D^{0} \rightarrow K^{-} \pi^{+} \pi^{0}$ and an inclusive search for $l \pi^{+}$
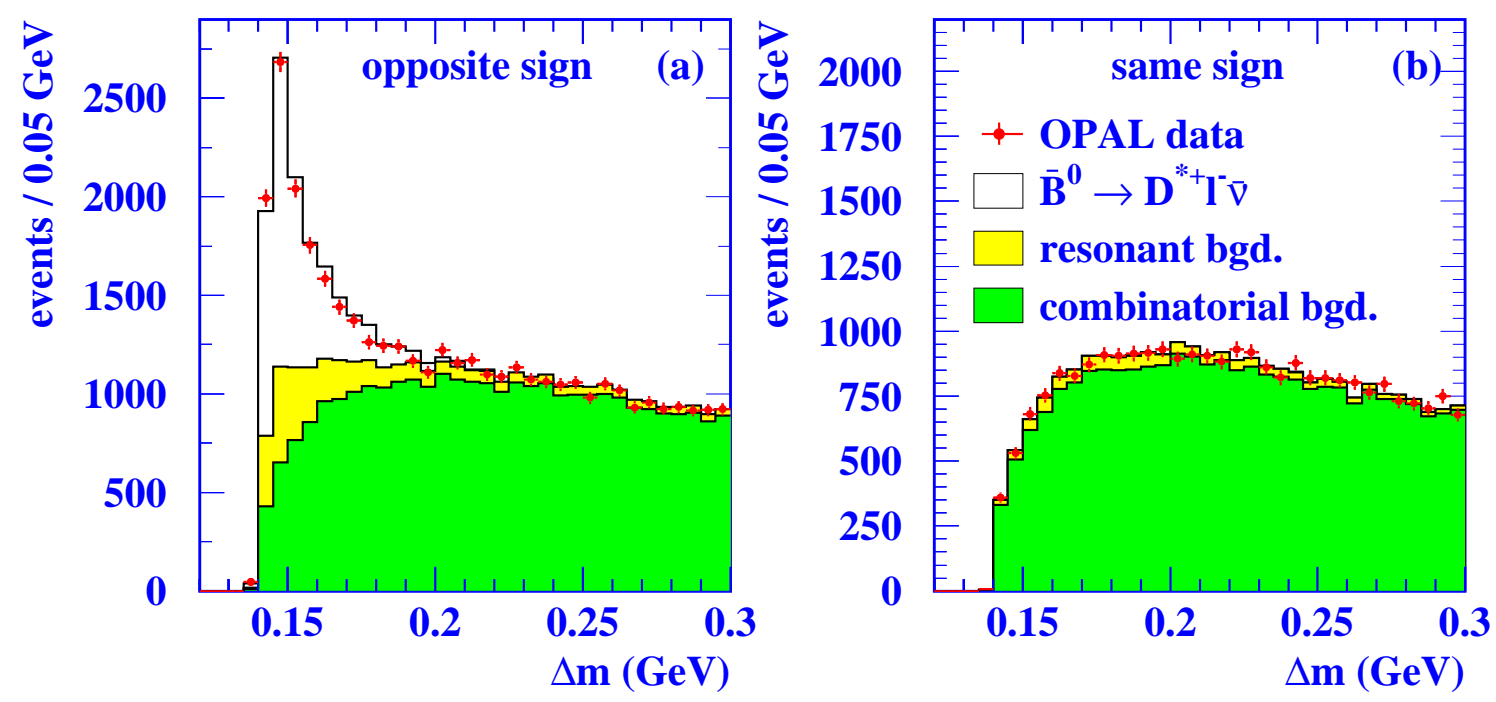


\section{Extracting $\omega$ distribution:}
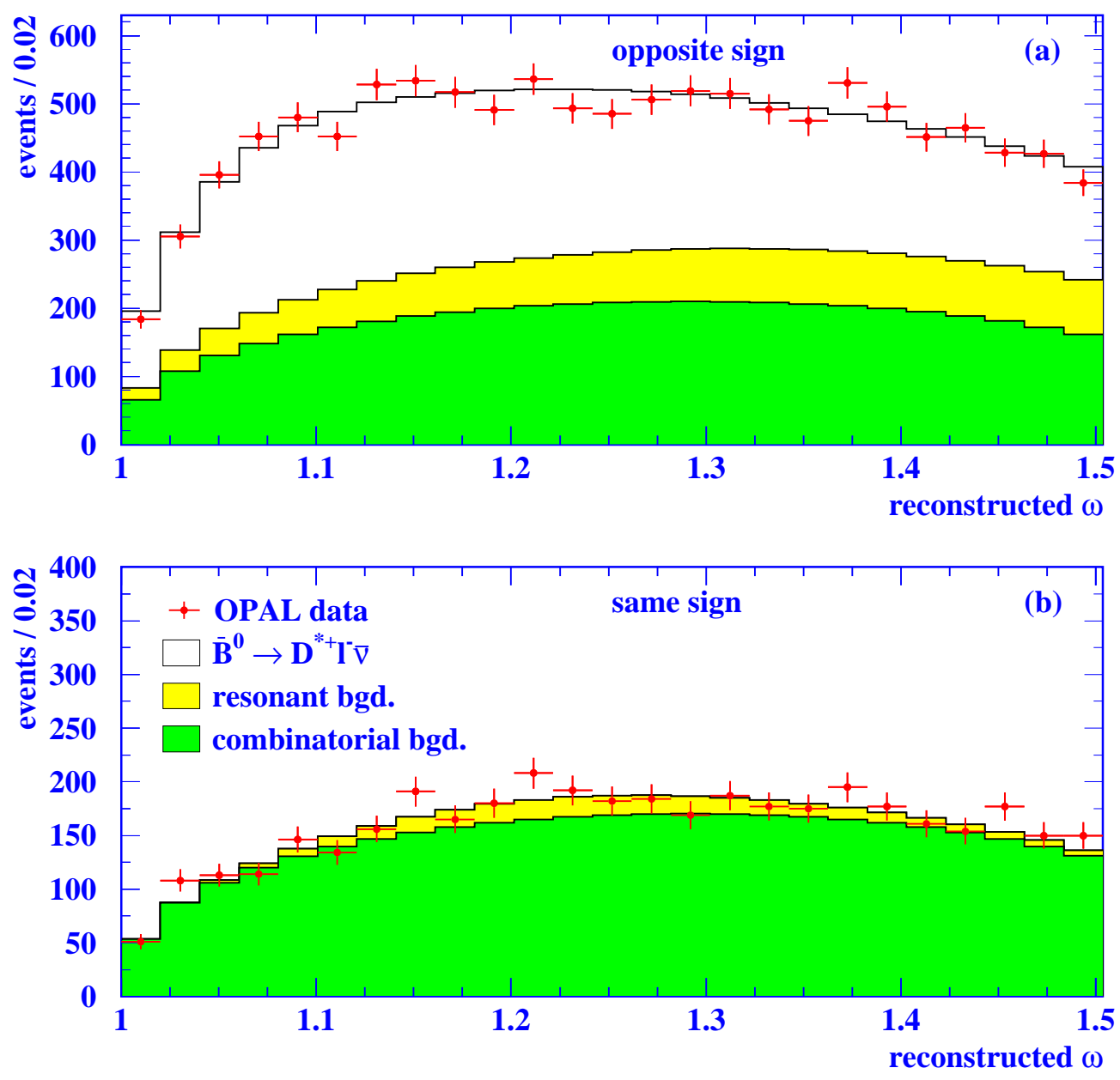

and finally:

$$
\left|V_{c b}\right|=(40.7 \pm 1.1 \pm 2.2 \pm 1.6) \times 10^{-3}
$$




\section{All exclusive results:}

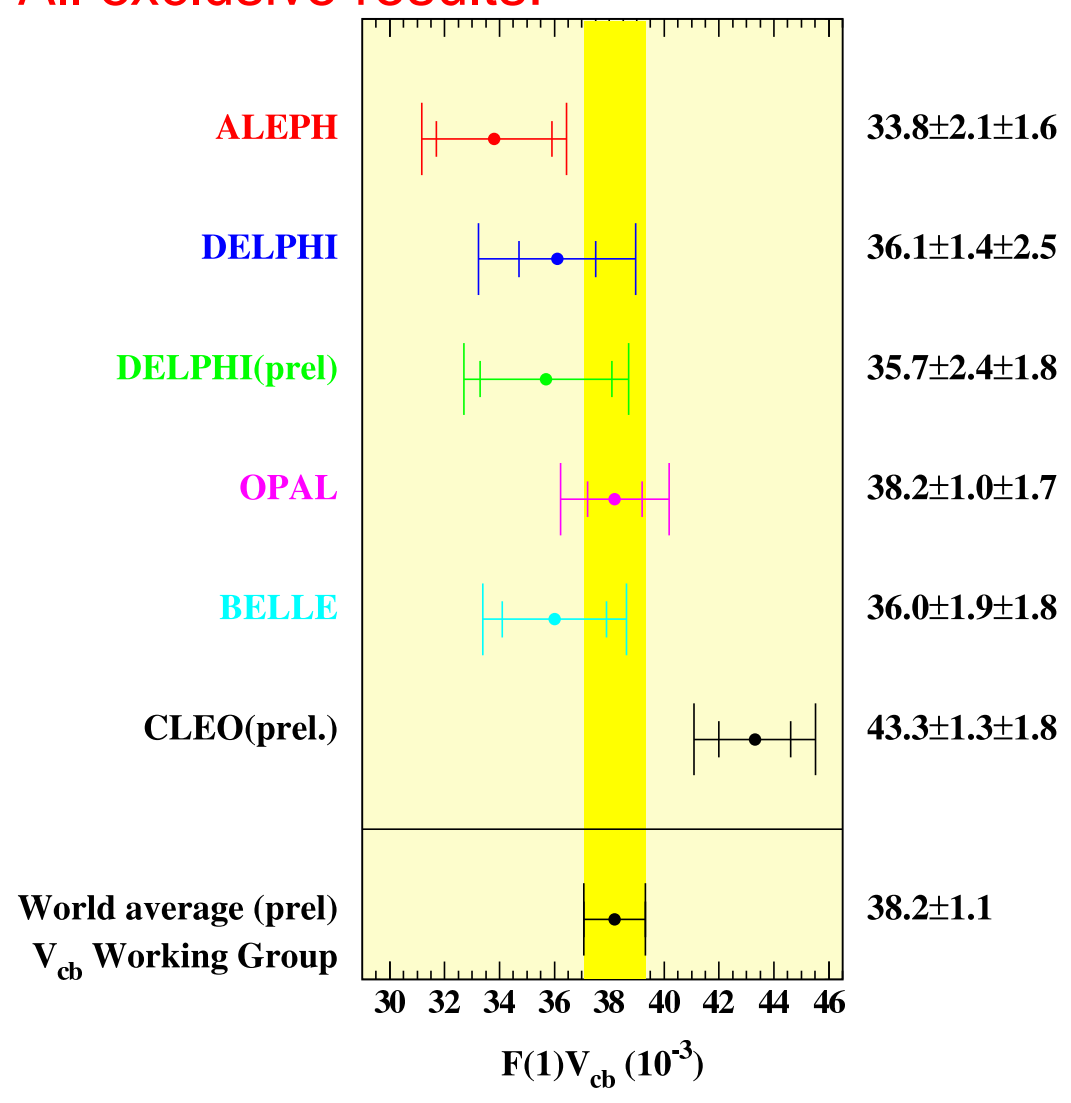


Method 2 - using inclusive $b \rightarrow c l \nu$

\section{- LEP measured $\operatorname{Br}(b \rightarrow X l \nu)=10.654 \pm 0.23 \%$}

- Subtract $\operatorname{Br}(b \rightarrow u l \nu)$

- Obtain $\operatorname{Br}(b \rightarrow c l v)$

- $\left|V_{c b}\right|=0.0411 \sqrt{\frac{1.55}{0.105} \Gamma(b \rightarrow c l \nu)}\left(1-0.024\left(\frac{\mu_{\pi}^{2}-0.5}{0.2}\right)\right)$

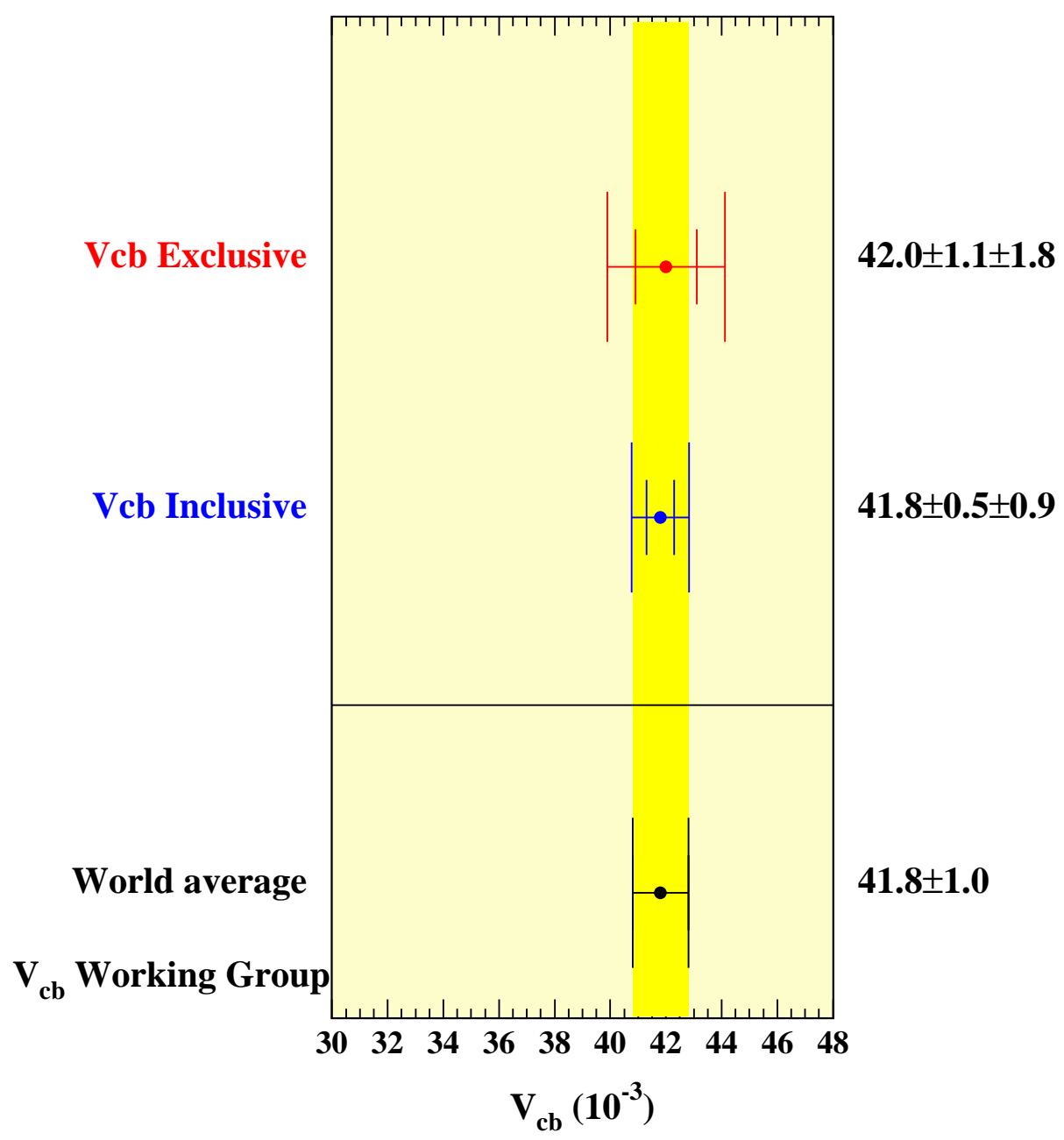


CKM summary

- $\left|V_{u b}\right|=4.09_{-0.69}^{+0.59} \times 10^{-3}$

- $\left|V_{c b}\right|=41.8 \pm 1.0 \times 10^{-3}$

- $\frac{\left|V_{u b}\right|}{\left|V_{c b}\right|}=0.103 \pm 0.022$ 


\section{B Oscillation}

How to Measure B Oscillation

- Find a $B^{0}$ (background)

- Measure the decay time (resolution)

- Find decay flavor $\left(B^{0}\right.$ or $\left.\bar{B}^{0}\right)$

- Find production flavor (mistag)

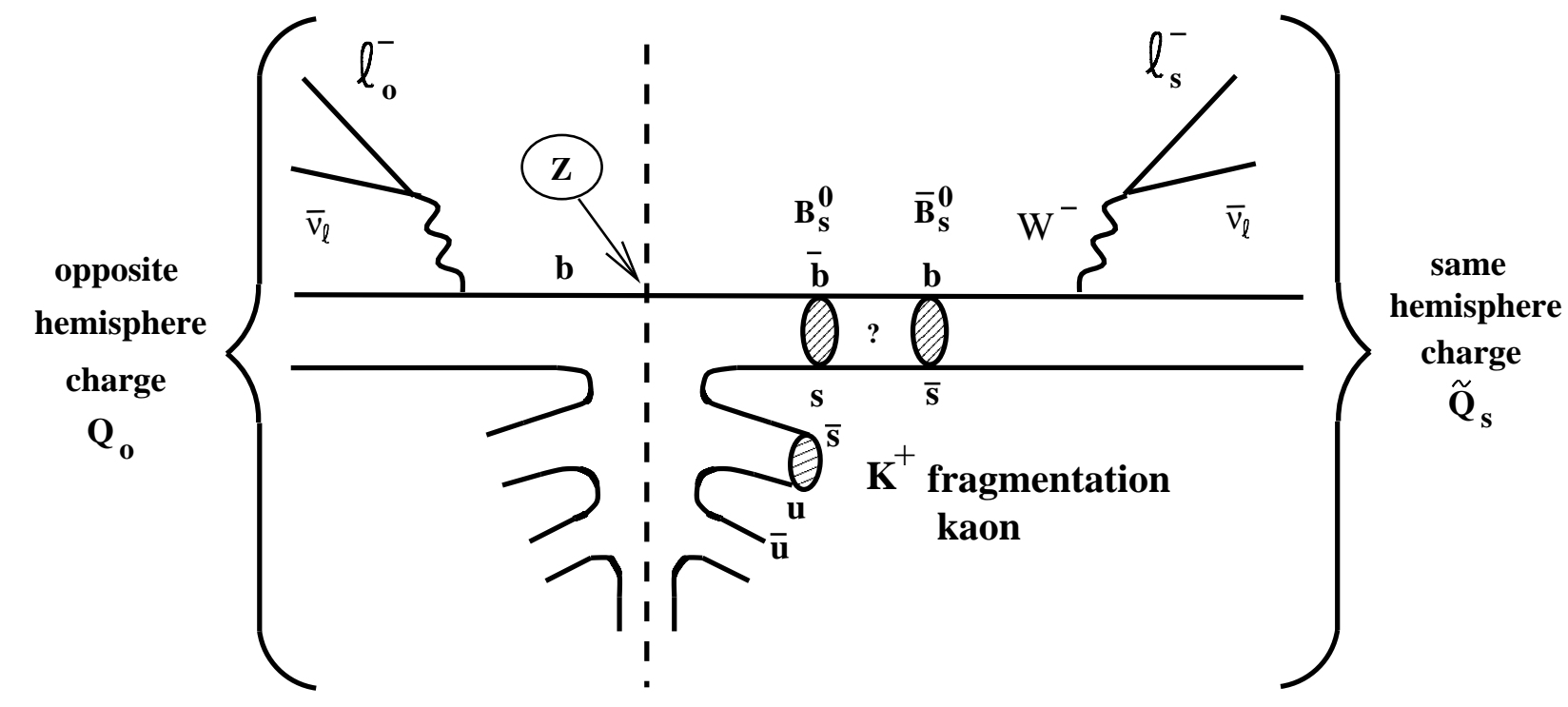


- Fit

$$
R(t)=\frac{N_{ \pm \pm}}{N_{ \pm \pm}+N_{+-}}=f+(1-2 f) \cdot \sin ^{2}\left(\frac{\Delta m \cdot t}{2}\right)
$$
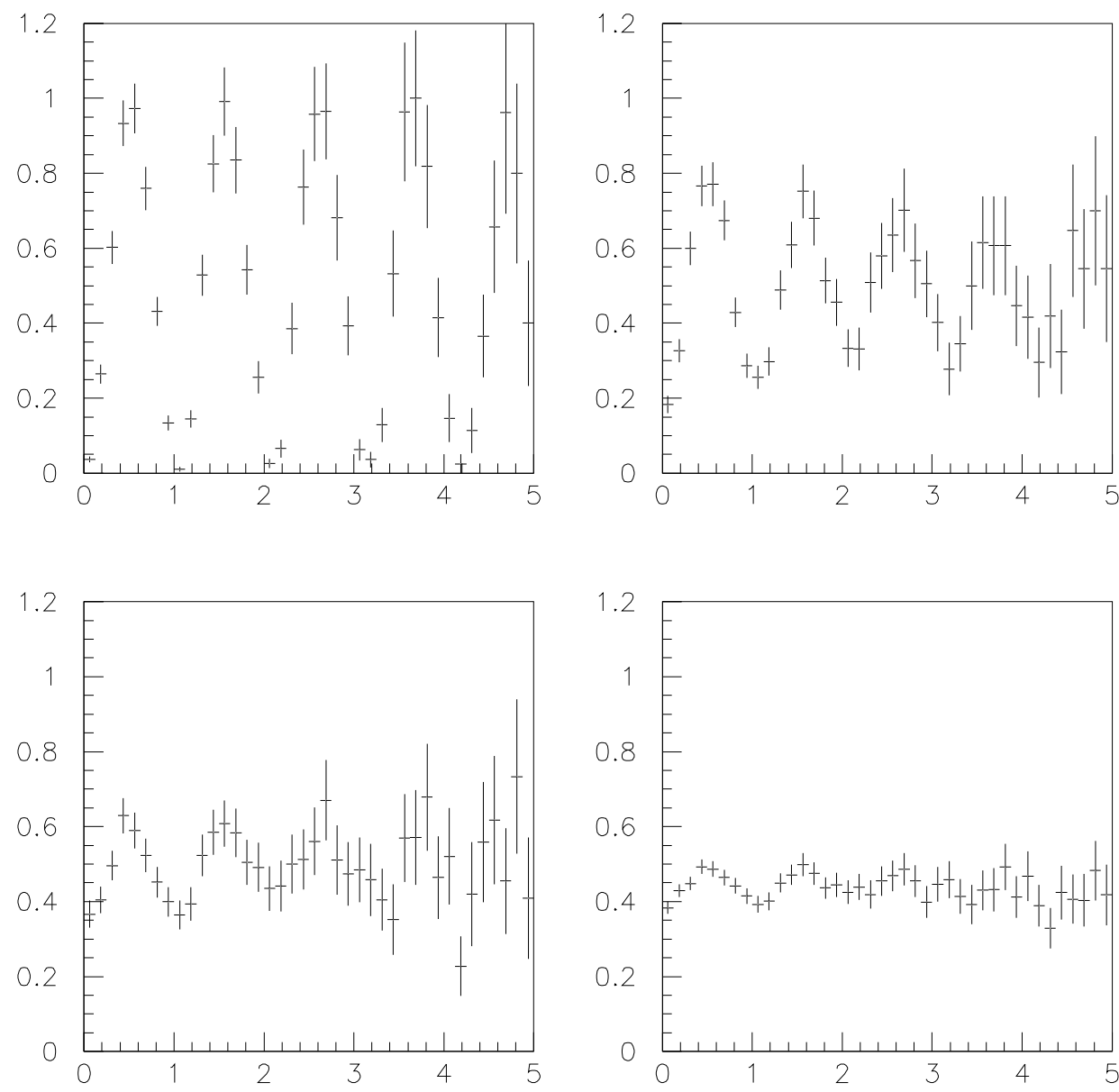


\section{$B_{d}^{0}$ mixing}

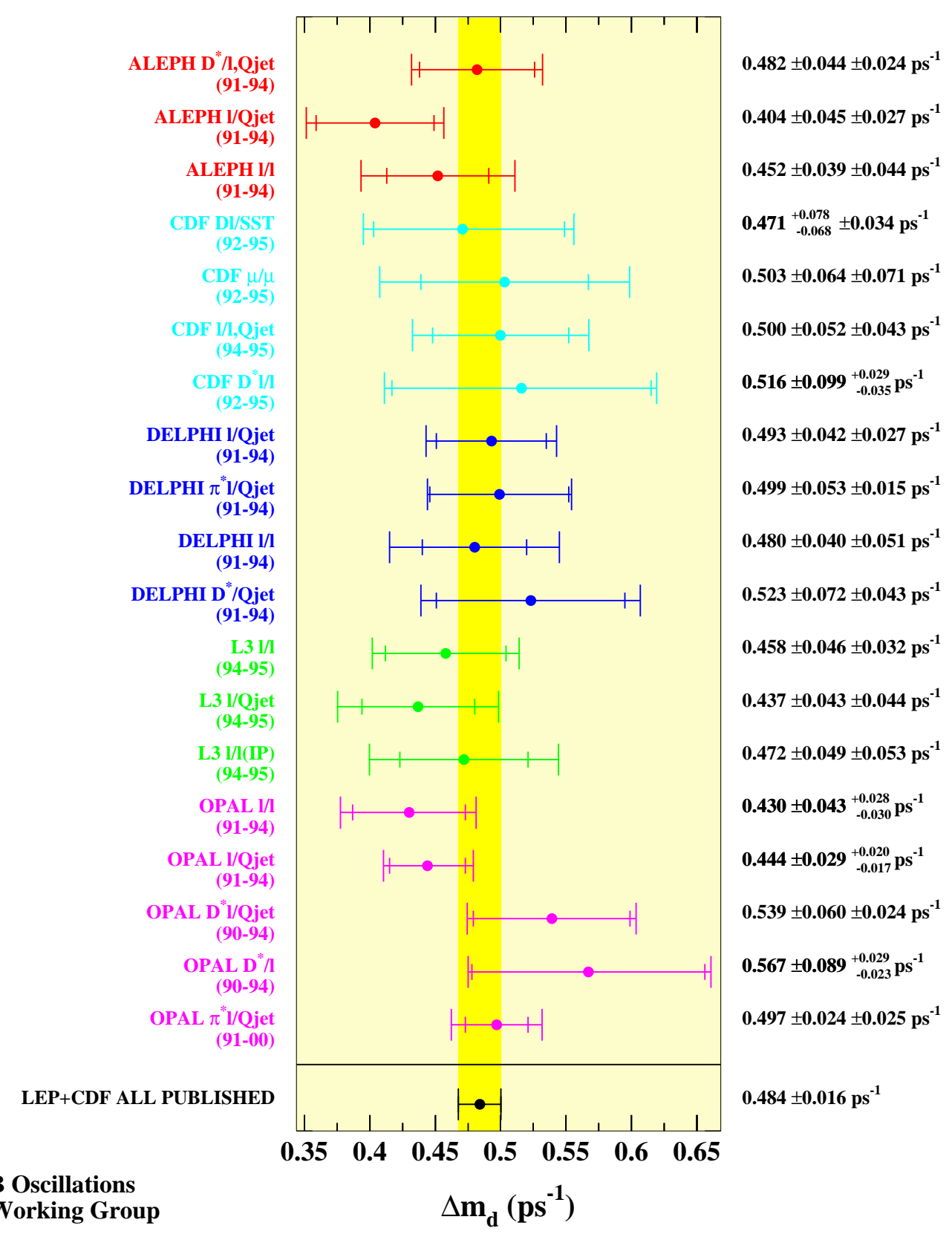




\section{Amplitude method}

- Substitute in likelihood function:

$$
1 \pm \cos \Delta m_{s} t \rightarrow 1 \pm A \cos \Delta m_{s} t
$$

- Fit amplitude A for fixed $\Delta m_{s}$.

- Expect $A=1$ for true value of $\Delta m_{s}$ and $A=0$

(far) below true $\Delta m_{s}$.

- Values of $\Delta m_{s}$ excluded ( $95 \%$ C.L.) if

$$
A+1.645 \sigma_{A}<1 \text {. }
$$

- Sensitivity is defined for $A+1.645 \sigma_{A}=1$.
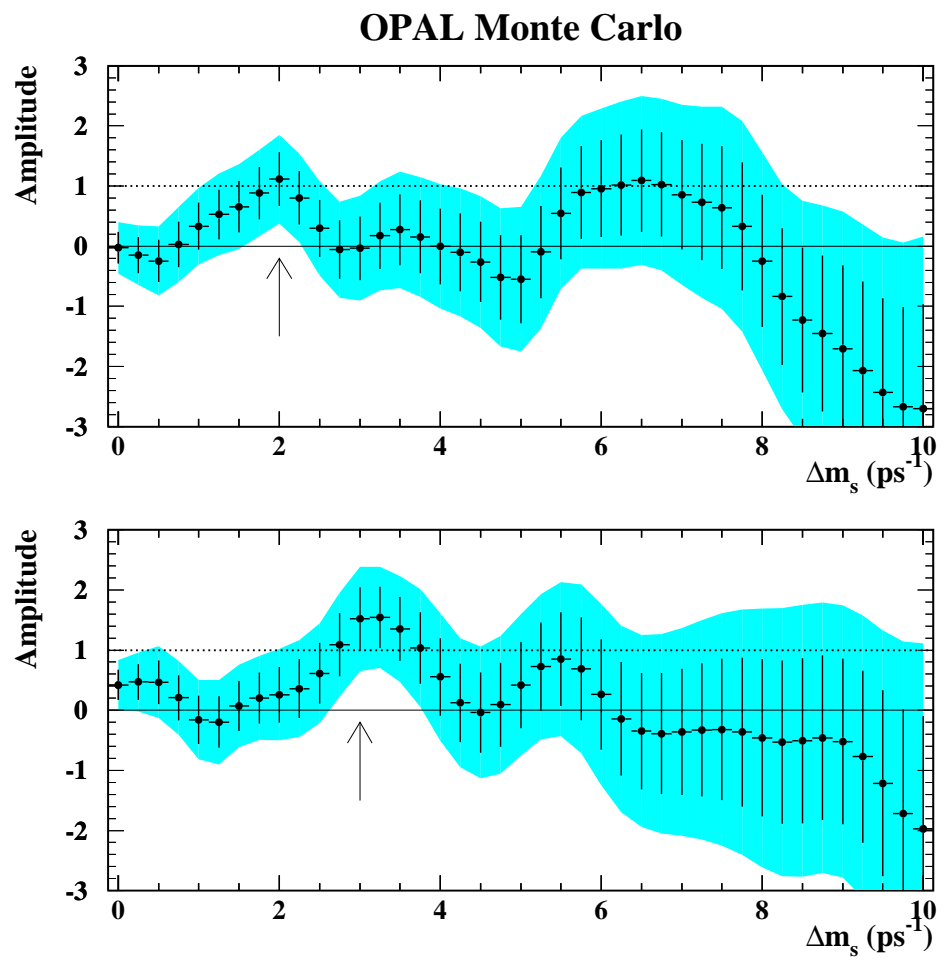


\section{Search Methods for B oscillation}

- Inclusive.

Eff. Purity Resolution

- Semi-Inclusive:

Single Lepton.

Dileptons.

- Semi-exclusive:

Lepton-kaon.

$\phi$ lepton.

Lepton+"D".

- Exclusive:

$D_{s}$-hadron.

$D_{s}$-lepton.
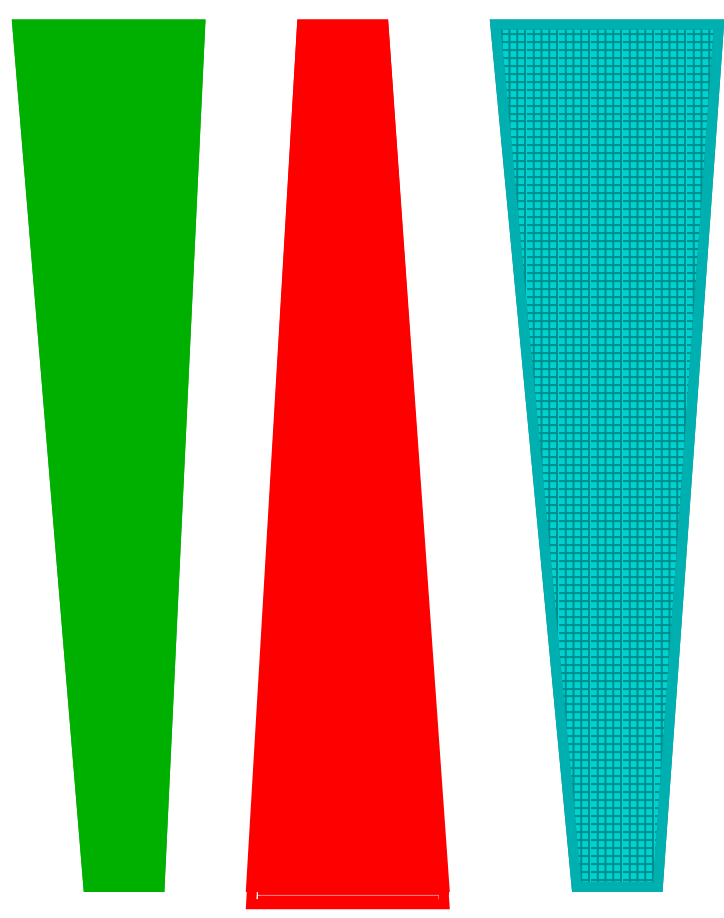


\section{Fully inclusive search - DELPHI}

- Event selection: soft leptons and inclusively reconstructed vertices $(120 \mathrm{~K} / 500 \mathrm{~K})$

- Decay time reconstruction:

1. $\mathrm{E} / \mathrm{p}$ constraint to rescale $\mathrm{B}$ momentum and estimate $\nu$ momentum - global fit using direction and mass constraint 2. Inclusive B reconstruction based on B track probability (NN)

- Decay flavor:

1. Lepton charge (special care for b/c origin due to softness)

2. For inc. vtx. use the dipole charge of charm/bottom tracks in the $B$ rest-frame.

- Production flavor: Jet-charge, leptons, kaon and protons.

- Result: $\Delta m_{s}>1.1 p^{-1}$

(sensitivity $6.1 \mathrm{ps}^{-1}$ ) 


\section{Inclusive lepton analysis - ALEPH}

- Event selection: High $p, p_{T}$ leptons.

- Decay time reconstruction:

charm vtx combined with the lepton for decay length and momentum is estimated with the addition of the hemisphere missing energy $(\nu)$

Decay time resolution $\approx 1 / 4-1 / 3 p s$

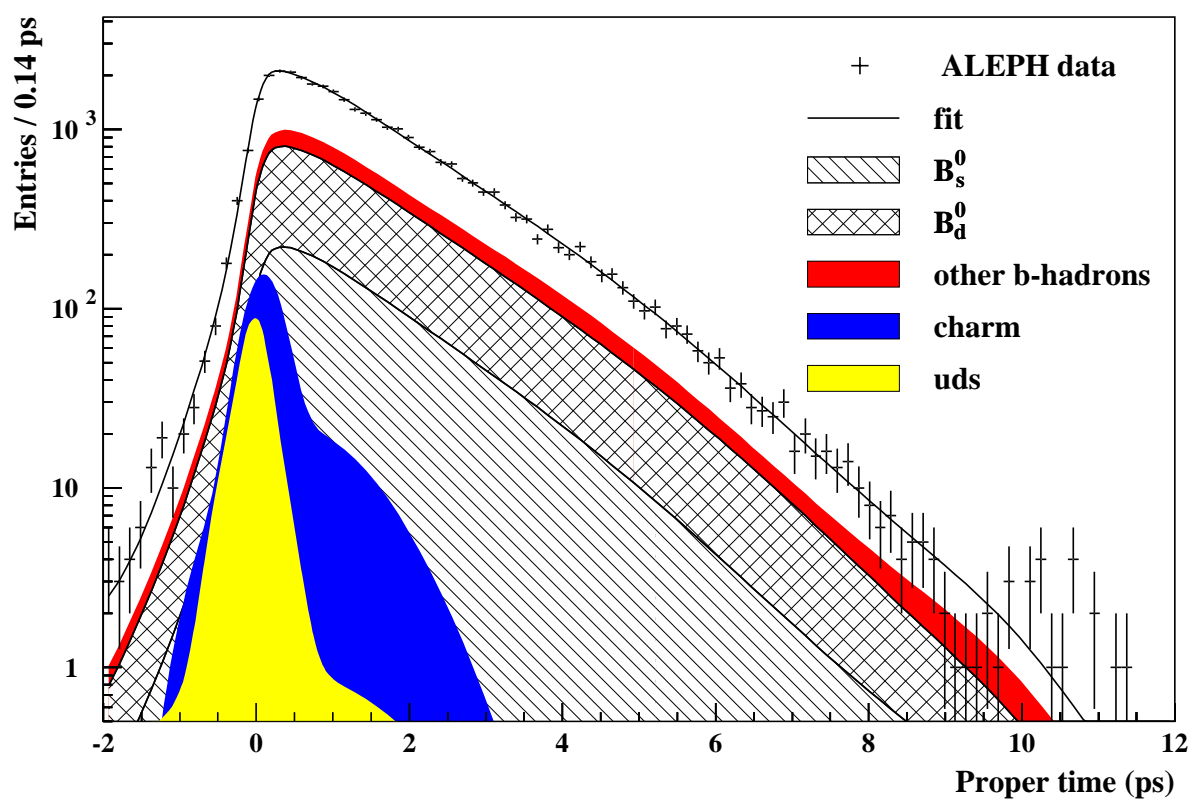


- Decay flavor: Directly from the lepton charge.

- Decay flavor: Opp. hem. lepton, frag. kaon, jet-charge.

- Result: $\Delta m_{s}>11.1 p s^{-1}$

(sensitivity $11.9 \mathrm{ps}^{-1}$ )

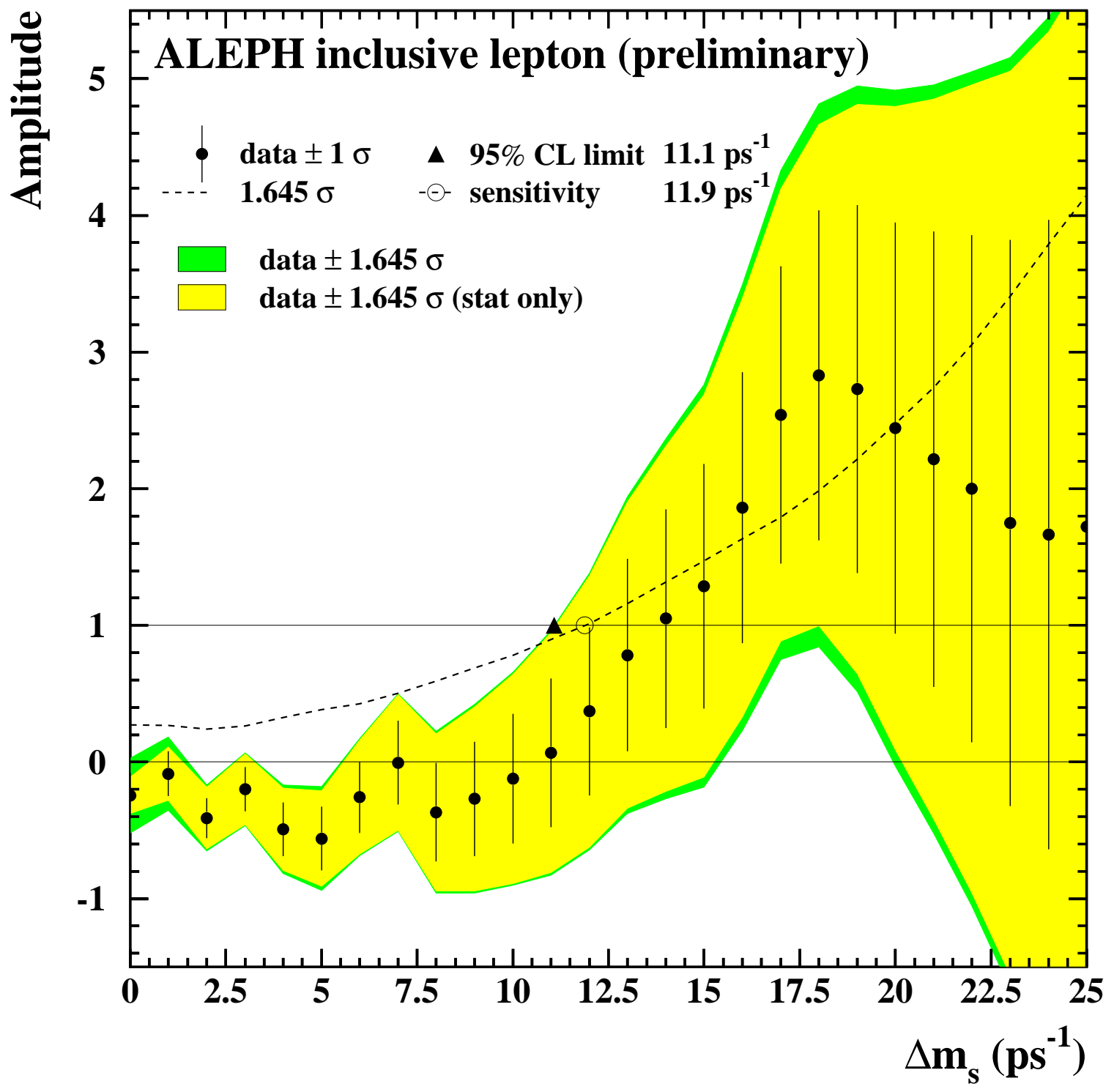




\section{SLD analyses}

- Much smaller Z sample (400K).

- Much better vtxing. $\rightarrow$ at best $\sigma_{t}=0.05 p s$

- Polarized electrons.

- Three final states: lepton + "D", $D_{s}+$ tracks, dipole analysis.

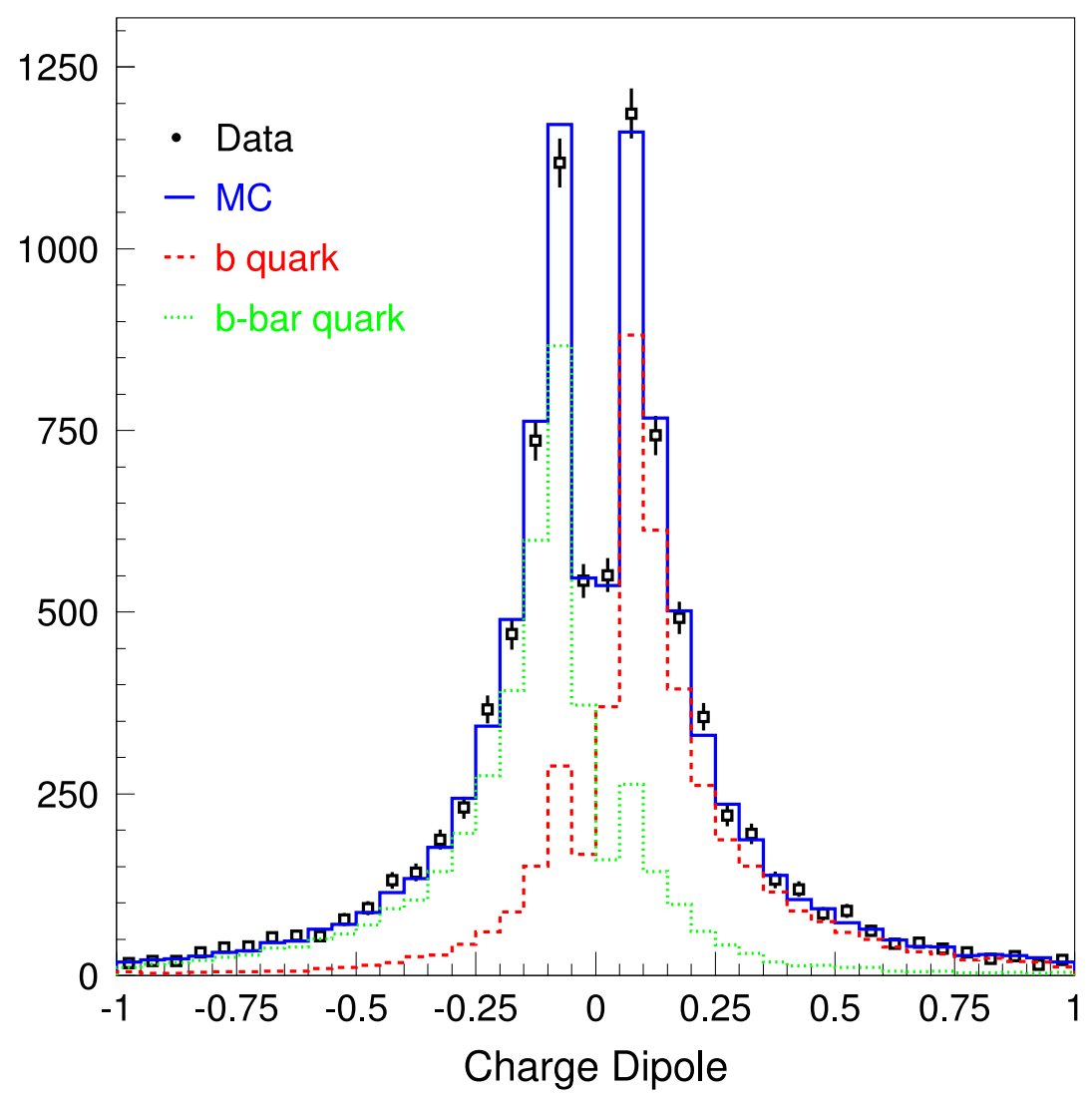


- Unique tag from FB asymmetry - 100\% efficient, $72 \%$ correct tag.

- Result: $\Delta m_{s}>7.6 p s^{-1}$

(sensitivity $13.0 \mathrm{ps}^{-1}$ )

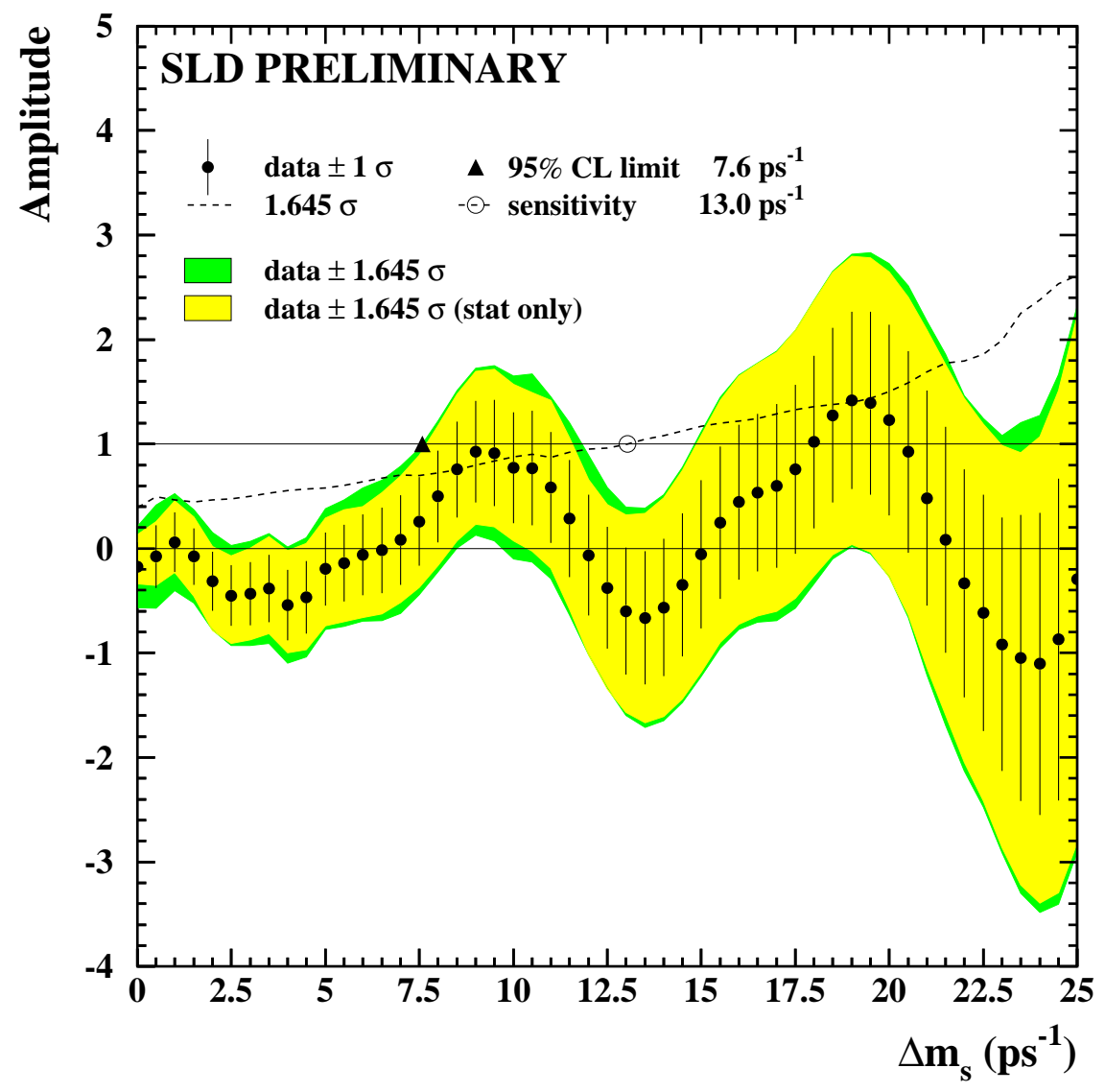




\section{Exclusive analysis from OPAL}

- Four decay channels of the $D_{s}$ :

$$
\begin{aligned}
& \mathrm{B}_{\mathrm{s}} \rightarrow \mathrm{D}_{\mathrm{s}} l^{+} \nu \\
& \rightarrow \mathrm{K}^{\star 0} \mathrm{~K}^{-}, \quad \mathrm{K}^{\star 0} \rightarrow \mathrm{K}^{+} \pi^{-} \\
& \rightarrow \phi \pi^{-}, \quad \phi \rightarrow \mathrm{K}^{+} \mathrm{K}^{-} \\
& \hookrightarrow K^{0} \mathrm{~K}^{-}, \quad K^{0} \rightarrow \pi^{+} \pi^{-} \\
& \hookrightarrow \phi l^{-} \bar{\nu} \mathrm{X}, \quad \phi \rightarrow \mathrm{K}^{+} \mathrm{K}^{-}
\end{aligned}
$$

OPAL
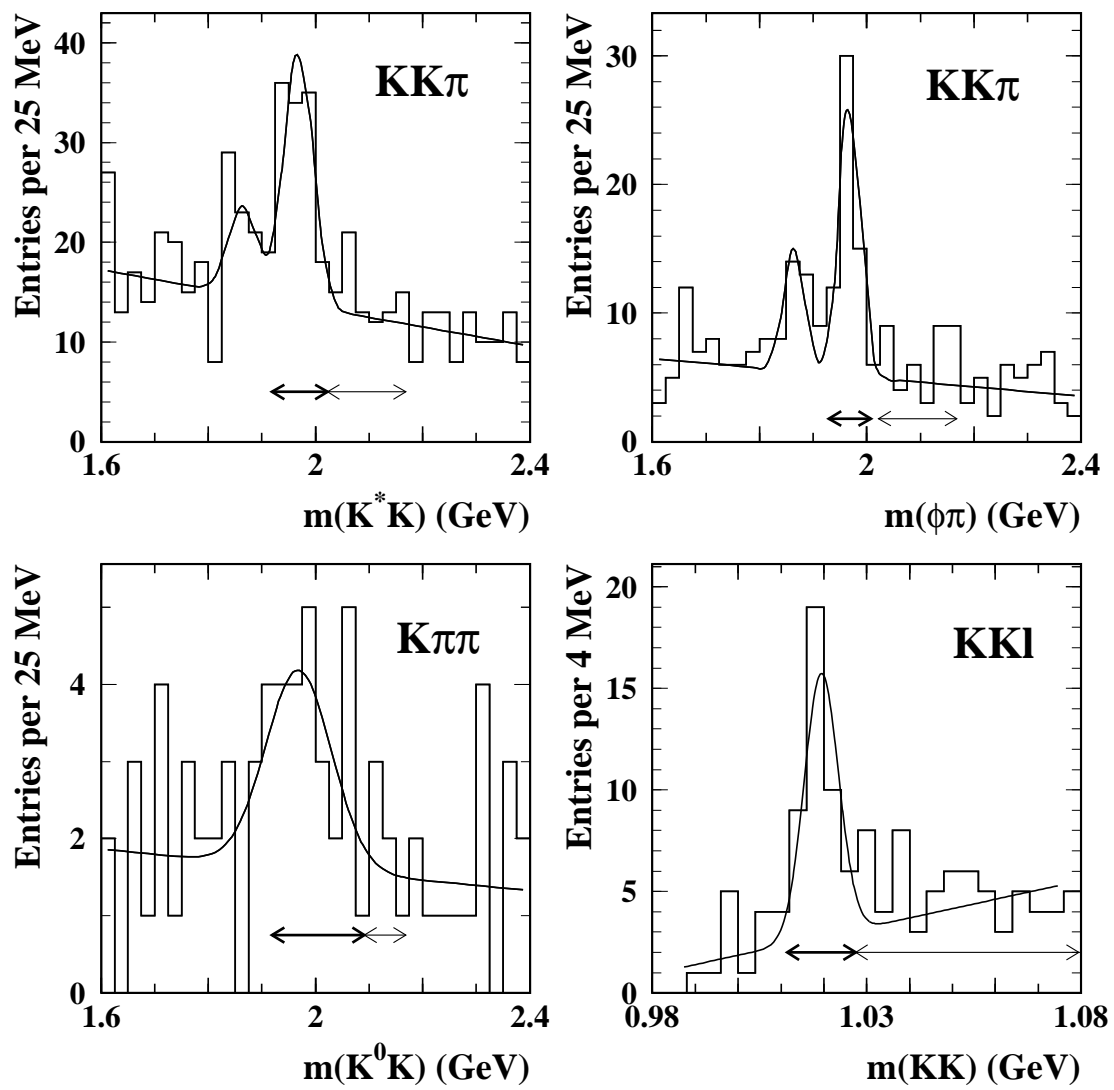

- Total of $116 \quad B_{s} \rightarrow D_{s} l$ events. 


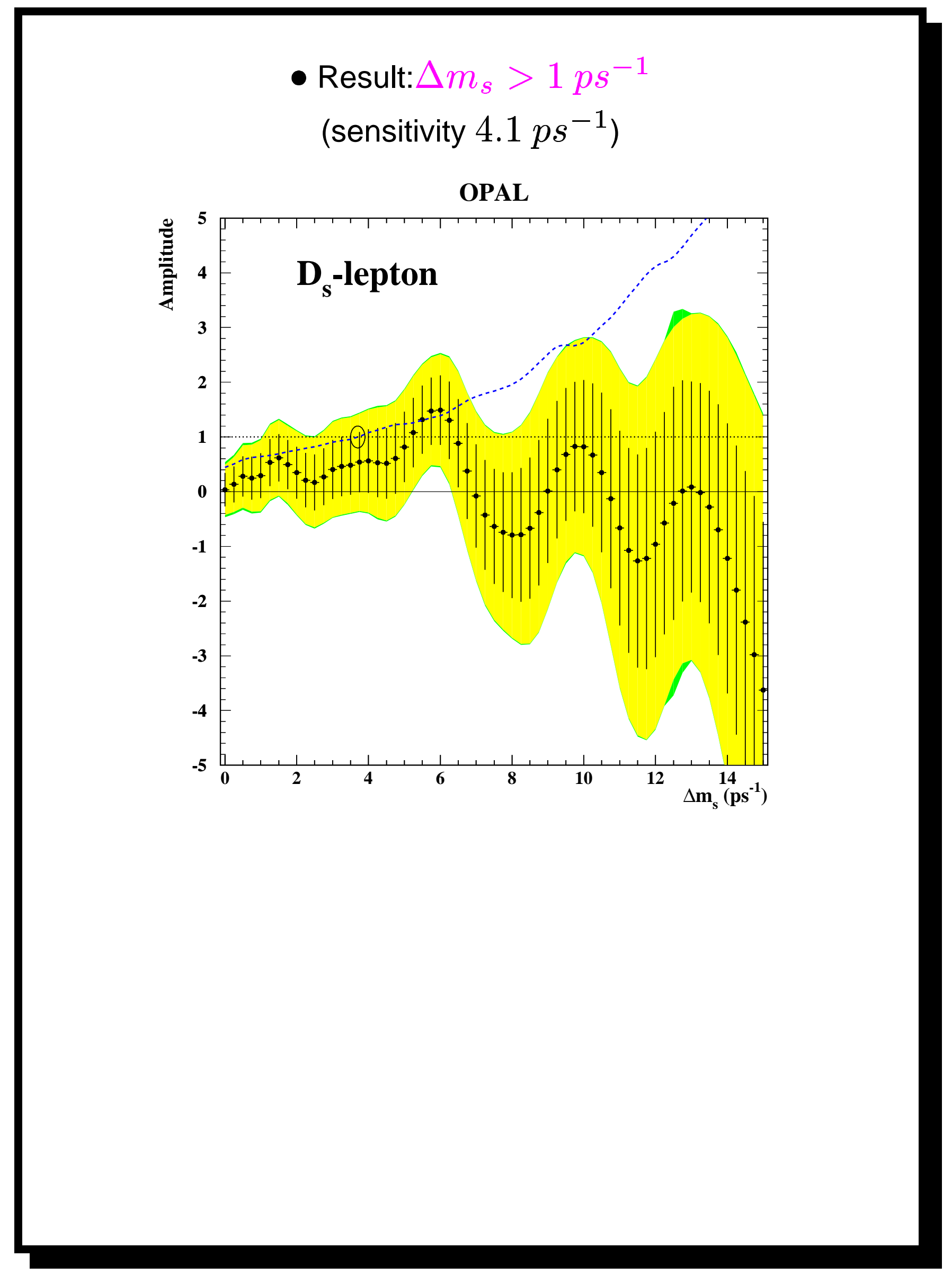




\section{$B_{s}$ Oscillation Summary}

- World Combined Result

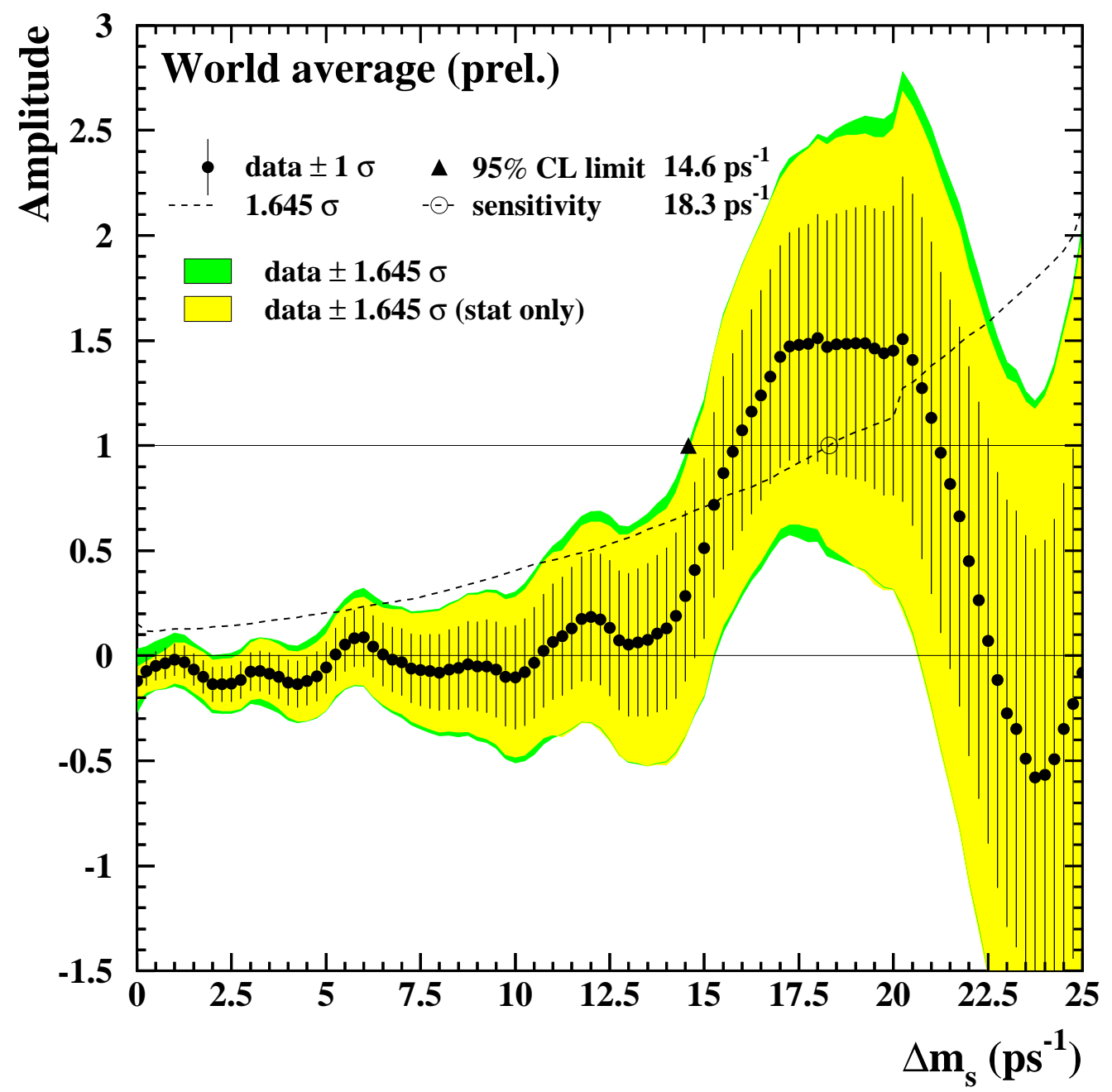

Limit at $14.6 \mathrm{ps}^{-1} \quad$ Sensitivity at $18.3 p s^{-1}$

- Is there a hint at $17 p s^{-1}$ ? 
ALEPH D h

(91-95)

ALEPH B +D I

(91-95, prel.)

ALEPH

(91-95, no D l, prel.)

CDF $I \phi / 1$

(92-95)

DELPHI B +D h

(92-95)

DELPHI D I+ $\phi$ I

(92-95 prel)

DELPHI I (92-95, prel)

DELPHI vtx

(92-95, prel)

OPAL I

(91-95)

OPAL D I

(91-95)

SLD l+D

(96-98, prel.)

SLD dipole

(96-98, prel.)

SLD D

(96-98, prel.)

World average (prel.)

B Oscillations

Working Group

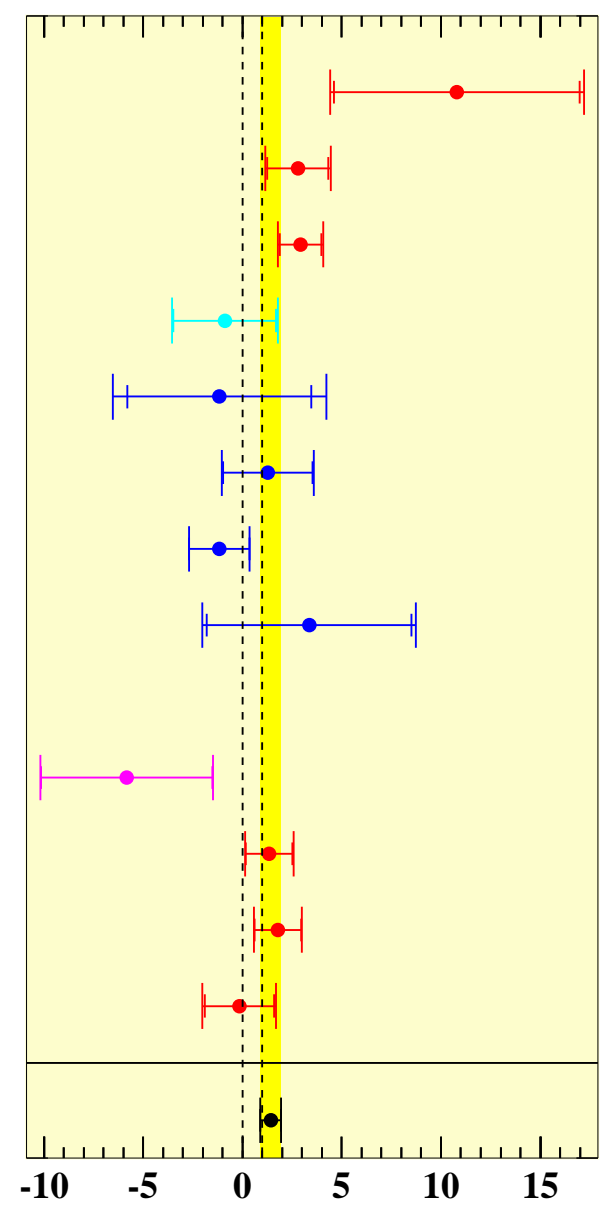

amplitude at $\Delta \mathrm{m}_{\mathrm{s}}=17.0 \mathrm{ps}^{-1}$ amplitude

(sensitivity)

$10.79 \pm 6.20_{-1.45}^{+1.65} \quad\left(4.1 \mathrm{ps}^{-1}\right)$

$2.79 \pm 1.54 \pm 0.62 \quad\left(7.4 \mathrm{ps}^{-1}\right)$

$2.92 \pm 1.05 \pm 0.47\left(11.7 \mathrm{ps}^{-1}\right)$

$-0.89 \pm 2.59_{-0.62}^{+0.62}\left(5.1 \mathrm{ps}^{-1}\right)$

$-1.16 \pm 4.63 \pm 2.75 \quad\left(3.2 \mathrm{ps}^{-1}\right)$

$1.28 \pm 2.26 \pm 0.50 \quad\left(8.7 \mathrm{ps}^{-1}\right)$

$-1.17 \pm 1.51 \pm 0.27 \quad\left(9.9 \mathrm{ps}^{-1}\right)$

$3.37 \pm 5.16 \pm 1.51 \quad\left(6.1 \mathrm{ps}^{-1}\right)$

not measured

$\left(7.2 \mathrm{ps}^{-1}\right)$

$-5.83 \pm 4.32 \underset{-0.58}{+0.51} \quad\left(4.2 \mathrm{ps}^{-1}\right)$

$1.34 \pm 1.18_{-0.24}^{+0.34} \quad\left(6.3 \mathrm{ps}^{-1}\right)$

$1.78 \pm 1.17_{-0.25}^{+0.21} \quad\left(8.6 \mathrm{ps}^{-1}\right)$

$\mathbf{- 0 . 1 6} \pm 1.74_{-0.69}^{+0.59} \quad\left(1.7 \mathrm{ps}^{-1}\right)$

$1.42 \pm 0.53 \quad\left(18.3 \mathrm{ps}^{-1}\right)$

- $2.6 \sigma$ effect.

- No (real) improvement is expected before the onset of $\mathrm{LHC}$

- $\mathrm{ATLAS} / \mathrm{CMS}$ sensitivity $\approx 38 p s^{-1}$ 


\section{Summary and Outlook}

- The LEP B program exceeded all expectations.

- $\sigma_{\tau_{B_{u}, B_{d}}} \approx 1 \%, \sigma_{\tau_{B_{s}, \Lambda_{b}}} \approx 4 \%$

- $\sigma_{V_{u b}}=16 \%$

- $\sigma_{V_{c b}}=2.4 \%$

- $\Delta m_{d}=0.484 \pm 0.016 p^{-1}$ (3.3\%)

- $\Delta m_{s}>14.6 p^{-1}$

- Comparing results from LEP and SLD, statistics .vs. resolution, SLD performs as well as the "big guys". Concluding that the key for measuring $\Delta m_{s}$ is in the resolution... 